\title{
Reduced expression and prognostic implication of inhibitor of growth 4 in human osteosarcoma
}

\author{
DAHANG ZHAO, XIANGJIE LIU, YUNGE ZHANG, ZHAOMING DING, \\ FENG DONG, HONGWEI XU, BAOXIN WANG and WENBO WANG
}

\begin{abstract}
The Third Department of Orthopedics, The First Affiliated Hospital of Harbin Medical University, Harbin, Heilongjiang 150001, P.R. China
\end{abstract}

Received January 14, 2015; Accepted February 16, 2016

DOI: $10.3892 / 01.2016 .4324$

\begin{abstract}
Osteosarcoma is the most prevalent type of primary malignant bone tumor. Inhibitor of growth 4 (ING4) has been demonstrated to function as a tumor suppressor through multiple pathways, and is its expression is understood to be suppressed or reduced in various malignancies. The present study aimed to investigate the expression of ING4 and to determine its prognostic value in osteosarcoma tissue. Formalin-fixed, paraffin-embedded tissue microarrays were analyzed, and contained 41 osteosarcoma specimens and 11 normal bone tissue specimens with duplicate cores. ING4 expression was evaluated by immunohistochemical staining. The association between ING4 expression in the osteosarcoma and normal bone tissues was analyzed, in addition to the association between ING4 expression and Enneking classification of the osteosarcoma tissues. A significant statistical difference was observed in the ING4 immunohistochemical staining score between the osteosarcoma and normal bone tissues $(\mathrm{P}<0.001)$. Furthermore, a significant negative correlation was detected between the ING4 immunohistochemical staining scores and the Enneking classification results of the 41 osteosarcoma tissues $(\mathrm{P}=0.002)$. Low expression of ING4 was observed in the osteosarcoma specimens, and this reduced expression of ING4 was negatively correlated with Enneking classification. Thus, the results of the present study indicate that ING4 may serve as a promising prognostic marker in osteosarcoma.
\end{abstract}

Correspondence to: Professor Wenbo Wang, The Third Department of Orthopedics, The First Affiliated Hospital of Harbin Medical University, 23 Youzheng Street, Harbin, Heilongjiang 150001, P.R. China

E-mail: wenbo-wang@hotmail.com

Abbreviations: ING4, inhibitor of growth 4; TMAs, tissue microarrays; NF- $\kappa \mathrm{B}$, nuclear factor $-\kappa \mathrm{B}$

Key words: inhibitor of growth 4, osteosarcoma, Enneking classification, prognosis

\section{Introduction}

Osteosarcoma, the most common primary bone malignancy, accounts for $20 \%$ of all bone tumors and $\sim 5 \%$ of all pediatric tumors (1). Osteosarcoma has an overwhelming tendency for invasion and early metastasis (2). Although treatment with surgery and neoadjuvant chemotherapy appears to cure 60-70\% of cases (3), the 5-year survival rate for patients with recurrent and metastatic osteosarcoma is only $20 \%(4,5)$. Research investigating the mechanism of osteosarcoma development has primarily focused on chromosomal abnormalities, genetic alterations of tumor suppressor genes, activation of oncogenes and dysregulation of major signaling pathways. However, the molecular events that lead to the development of osteosarcoma are not yet fully understood.

Inhibitor of growth 4 (ING4) functions as a tumor suppressor, thus serving an inhibitory role during the generation and development of various types of tumor, including thyroid (6), gastric (7), lung (8) and breast cancer (9). ING4 physically interacts with and phosphorylates $\mathrm{p} 65$, a subunit of nuclear factor- $\kappa \mathrm{B}(\mathrm{NF}-\kappa \mathrm{B})$, therefore suppressing the activity of NF- $\kappa B$ (10). The inhibition of NF- $\kappa B$ negatively regulates various target genes, including matrix metalloproteinase (MMP)-2, MMP-9, interleukin (IL)-6, IL-8, cyclooxygenase-2 and colony stimulating factor-3. The downregulation of these cytokines inhibits angiogenesis and tumor cell growth (11-15). In RKO colorectal cancer cells, ING4 expression was able to decrease the cell population in the S-phase of the cell cycle in a p53-dependent manner, as well as upregulate p21 expression (16). In addition, ING4 may induce $\mathrm{G}_{2} / \mathrm{M}$ cell cycle arrest, and enhance chemosensitivity to etoposide and doxorubicin in HepG2 hepatocellular carcinoma cells (17). In general, there are two regulatory mechanisms of the cell cycle: p21 and Bcl-2-associated X protein (Bax). ING4 upregulates p21 and Bax in a p53-independent manner. The upregulation of $\mathrm{p} 21$ enhances its binding to cyclin $\mathrm{B} 1$, while the compound of cyclin $\mathrm{B} 1 /$ cyclin-dependent kinase 1 is crucial for progression in the cell cycle (18). Conversely, in the p53-defective SaoS-2 cell line, ING4 cannot upregulate p21 or Bax, and is therefore suspected to regulate the cell cycle by directly interacting with p300 or p65 (19). Furthermore, ING4 is able to upregulate Bax while downregulating B cell lymphoma-2 (Bcl-2), thus, decreasing the ratio of $\mathrm{Bcl}-2 / \mathrm{Bax}$, resulting in cytochrome $c$ 
release from the mitochondrion and activation of caspase-3. Activated caspase-3 disrupts the function of poly(ADP-ribose) polymerase and, as a result, DNA cannot be repaired. Therefore, ING4 may also induce apoptosis through activation of the mitochondrial apoptotic pathway in a p53-independent manner (20-22). Evidence suggests that ING4 may suppress the expression of the target gene, hypoxia-inducible factor-1 $\alpha$, by interacting with its plant homeodomain finger motif under hypoxic conditions (23-25). In addition, ING4 serves a role in the suppression of loss of contact inhibition elicited by the overexpression of the proto-oncogene Myc or MYCN; however, ING4 cannot suppress the transcriptional activity of the proto-oncogene (26). Furthermore, ING4 is able to interact with liprin $\alpha-1$, a novel ING4-associated protein, to prevent invasion and metastasis (27).

ING4 expression is decreased in a number of tumor tissues and the degree of low expression corresponds with tumor grade $(11,18,20,28-31)$. Allelic loss and mutation of ING4 have also been observed in various cancer cell lines $(26,32,33)$, thus suggesting that ING4 is a promising tumor suppressor. Despite this, there are currently no previously published studies that have investigated ING4 expression in osteosarcoma tissues. Therefore, the exact role of ING4 in the tumorigenesis and progression of osteosarcoma has yet to be established. In the present study, the expression of ING4 was analyzed in osteosarcoma tissue, and associations between the expression of the ING4 protein and several clinical characteristics were evaluated.

\section{Materials and methods}

Materials. The present study was approved by the Ethics Commitee of The First Affiliated Hospital of Harbin Medical University (Harbin, China; approval no., HYDEC-D-2016-051) and was conducted according to the Declaration of Helsinki Principles (34). Written informed consent was obtained from the patients or their families for the publication of this study. The tissue microarrays (TMAs) used for this study were purchased from Ailina Xi'an Biological Technology Co., Ltd. (Xi'an, China). The OS804 osteosarcoma tissue microarray, containing 40 cases of osteosarcoma, and the $\mathrm{BO} 244 \mathrm{~b}$ tissue microarray, containing 1 case of osteosarcoma and 11 cases of normal bone tissue (in the absence of any other bony disease), were obtained during trauma surgery and each contained duplicate cores. All primary osteosarcoma biopsy specimens were obtained at the time of diagnosis, prior to any chemotherapy treatment, and were assessed according to the Enneking classification for malignant bone tumors $(35,36)$. The tissues were formalin-fixed (Sigma-Aldrich, St. Louis, MO, USA) and paraffin-embedded (Sigma-Aldrich), and cut into $5-\mu \mathrm{m}$ thick sections with $1.5-\mathrm{mm}$ individual cores. The array sections were mounted on positively-charged, super plus glass slides (Sigma-Aldrich). The primary antibody, mouse anti-human ING4 polyclonal antibody ( $3 \mu \mathrm{g} / \mathrm{ml}$; catalog no., ab90551), was purchased from Abcam (Cambridge, UK). The EliVision ${ }^{\mathrm{TM}}$ Plus kit (catalog no., KIT-9902) and 3,3'-diaminobenzidine (DAB; catalog no., DAB-0031/1031), which was used as substrate chromogen, were purchased from Fuzhou Maixin Biotech Co., Ltd. (Fuzhou, China).
Immunohistochemistry. Normal bone tissues $(\mathrm{n}=11)$ were selected as the positive control and the primary antibody was replaced with phosphate-buffered saline (PBS; Sigma-Aldrich) to function as the negative control. The expression of ING4 protein was analyzed by immunohistochemistry. Sections were baked for $30 \mathrm{~min}$ at $60^{\circ} \mathrm{C}$, and were subsequently deparaffinized by washing with xylene (Sigma-Aldrich) and rehydrated in a graded alcohol series. Antigen retrieval was performed using $1 \mathrm{X}$ antigen retrieval solution (catalog no., 03690; Sigma-Aldrich) for $2 \mathrm{~min}$ in a pressure cooker at boiling point, as observed by steaming. The sections were cooled to room temperature, washed three times in PBS for $5 \mathrm{~min}$, incubated for $10 \mathrm{~min}$ in $3 \%$ hydrogen peroxide (Sigma-Aldrich) at room temperature and washed again three times in PBS for $5 \mathrm{~min}$. Subsequently, the sections were incubated at $4{ }^{\circ} \mathrm{C}$ overnight with the primary antibody, diluted to 1:100 in antibody diluent (Sigma-Aldrich) solution. The slides were washed three times in $0.01 \%$ Tween-20 (Sigma-Aldrich) PBS for $5 \mathrm{~min}$, incubated for $20 \mathrm{~min}$ with polymer enhancer (Sigma-Aldrich) at room temperature, washed again three times with $0.1 \%$ Tween-20 PBS for 5 min and incubated for $30 \mathrm{~min}$ at room temperature with polymerized horseradish peroxidase anti-mouse immunoglobulin $\mathrm{G}$ (catalog no., ZB-2305; dilution, 1:500; ZSGB-Bio, Beijing, China). The tissues were subsequently incubated in peroxidase substrate DAB solution until the desired stain intensity had developed and rinsed in tap water. Finally, the slides were counterstained with Hematoxylin QS (catalog no., H-3404; Vector Laboratories, Inc., Burlingame, CA, USA), and cleared and mounted with permanent mounting medium (Vector Laboratories, Inc.).

Evaluation and scoring of the TMAs. A semiquantitative scoring system was used to evaluate theimmunohistochemistry results of ING4 protein localized to the nucleus. Immunoreactivity was assessed by the percentage of positive cells and the strength of staining. The percentage of positive cells was scored as follows: $1,<11 \% ; 2,11-50 \% ; 3,51-75 \%$; and $4,>75 \%$. The strength of staining was scored as follows: No staining, 0; light brown, 1; brown, 2; and dark brown, 3. The final score was determined by multiplying the proportion of positive cells score with the strength of staining score. Final scores were classified as follows: $<3,(-) ; 3-5,(+) ; 6-9,(++)$; and $>9,(+++)(21)$. A final score of $(-)$ and $(+)$ represented low expression, whilst $(++)$ and $(+++)$ represented high expression. All cases, including 41 osteosarcoma cases and 11 normal bone cases, were evaluated by three independent, blinded observers simultaneously, and a consensus score was established for each core. All sections were observed under an optical microscope (TCS NT; Leica Microsystems, Wetzlar, Germany).

Clinical outcome measures of the osteosarcoma specimens. The Enneking classification system for malignant bone tumors is based on considerations of grade and metastasis $(35,36)$. The stages of osteosarcoma are divided into I, II and III based upon the compartmentalization of the lesion. Stage I comprises of low grade lesions without metastases; stage II comprises of high grade lesions without metastases; and stage III comprises low or high grade lesions with metastases. 
A
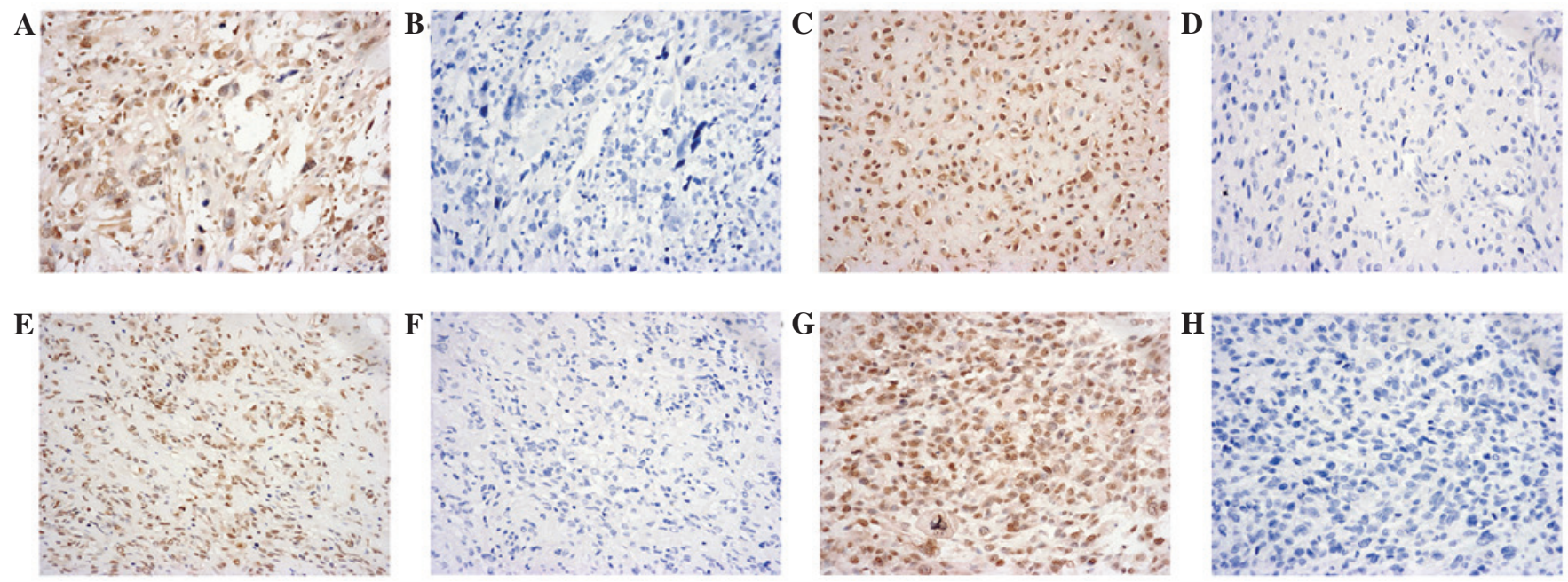

Figure 1. Immunohistochemical expression of ING4 protein in representative osteosarcoma tissues. (A) Immunohistochemical staining score of (-) obtained from a 27-year-old male with osteosarcoma located in the distal femur. (B) Negative control of A. (C) Immunohistochemical staining score of (+) obtained from a 19-year-old male with osteosarcoma located in the proximal tibia. (D) Negative control of C. (E) Immunohistochemical staining score of (++) obtained from a 15-year-old female with osteosarcoma located in the distal femur. (F) Negative control of E. (G) Immunohistochemical staining score of (+++) obtained from a 13-year-old male with osteosarcoma located in the distal femur. (H) Negative control of G. Magnification, x200. 3,3'-diaminobenzidine and hematoxylin QS staining.
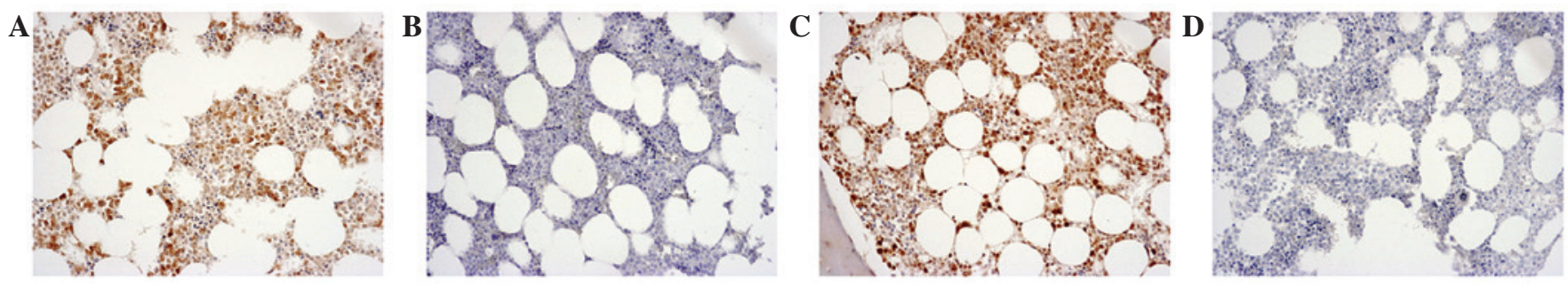

Figure 2. Immunohistochemical expression of ING4 protein in representative normal bone tissues. (A) Immunohistochemical staining score of (++) obtained from the normal bone of a 66-year-old female with a femur fracture. (B) Negative control of A. (C) Immunohistochemical staining score of (+++) obtained from the normal bone of a 35-year-old male with a tibia fracture. (D) Negative control of C. Magnification, x200. 3,3'-diaminobenzidine and hematoxylin QS staining.

Stages I and II are further subdivided as intracompartmental (A) or extracompartmental (B). Thus, all the specimens used in the present study were grouped as IA, IB, IIA, IIB or III.

Statistical analysis. The Mann-Whitney U test was used to analyze the diversity in the expression of ING4 between the osteosarcoma and bone tissue specimens. In addition, all clinicopathological variables were analyzed in association with the ING4 immunohistochemical score using univariate logistic regression analysis modeling. For statistical analysis of the ordinal data, gender was categorized as male or female; age at diagnosis as $<30$ or $\geq 30$ years; anatomical site as distal femur, proximal tibia or other; histological type as osteoblastic or other; and Enneking classification as IA, IB, IIA, IIB or III. Data was presented as the mean \pm standard error of the mean. SPSS version 19.0 software (SPSS, Inc., Chicago, IL, USA) was used to perform the statistical analyses, and $\mathrm{P}<0.05$ was considered to indicate a statistically significant difference.

\section{Results}

The mean age of the 41 patients with osteosarcoma ( 28 men and 13 women) was 31 years (range, 11-64 years). The anatomical sites of the primary tumor were the distal femur $(n=27)$, proximal tibia $(n=6)$, humerus $(n=3)$, scapula $(n=2)$, rib $(n=2)$ and fibula $(n=1)$. The histological type of the 41 specimens included osteoblastic $(n=26)$, chondroblastic $(n=7)$, fibroblastic $(n=4)$, periosteal $(n=3)$ and telangiectatic $(n=1)$. Metastatic lesions were identified in only 1 patient. Patient survival information was not obtained. The mean age of the 11 control cases, including 9 men and 2 women, was 59 years at the time when normal bone tissue was obtained (range, 41-75 years). The trauma sites of the normal bone tissues consisted of the femur $(n=5)$, the femoral head $(n=3)$, tibia $(n=2)$ and humerus $(n=1)$.

Each duplicated core of the 41 osteosarcoma specimens and 11 normal bone tissues had the same immunohistochemical staining score. Representative immunohistochemical staining results for the osteosarcoma tissues are presented in Fig. 1. A final score of (-) was observed in 7 (17.1\%) osteosarcoma specimens (Fig. 1A), a score of (+) in 19 (46.3\%) specimens (Fig. 1C), a score of (++) in $11(26.8 \%)$ specimens (Fig. 1E) and a score of (+++) in $4(9.8 \%)$ specimens (Fig. 1G). Negative controls were used for all osteosarcoma specimens (Fig. 1B, D, F and H). Representative immunohistochemical staining results for the normal bone tissues are presented in Fig. 2. A score of $(++)$ was observed in 
Table I. Comparison of ING4 expression between osteosarcoma and normal bone tissue specimens.

\begin{tabular}{lrrrrrrrr}
\hline & \multicolumn{5}{c}{ Immunohistochemical score } & & & \\
\cline { 2 - 5 } Group & - & + & ++ & +++ & Mean rank & U-value & P-value \\
\hline Osteosarcoma & 7 & 19 & 11 & 4 & 21.90 & & \\
Normal bone & 0 & 0 & 2 & 9 & 43.64 & & $<0.001$ \\
Total & 7 & 19 & 13 & 13 & & 37.00 & $<$ \\
\hline
\end{tabular}

A significant statistical difference was observed for the immunohistochemical staining score of ING4 between these osteosarcoma and normal bone tissue specimens. ING4, inhibitor of growth 4.

Table II. Correlation between clinicopathological variables and immunohistochemical staining score of ING4 in the osteosarcoma tissue specimens.

\begin{tabular}{|c|c|c|c|}
\hline \multirow[b]{2}{*}{ Characteristic } & \multicolumn{2}{|c|}{ Immunohistochemical score } & \multirow[b]{2}{*}{ P-value } \\
\hline & Low $(\mathrm{n}=26)$ & High $(n=15)$ & \\
\hline Gender & & & 0.389 \\
\hline Male & 19 & 9 & \\
\hline Female & 7 & 6 & \\
\hline Age at diagnosis, years & & & 0.923 \\
\hline$<30$ & 10 & 6 & \\
\hline$\geq 30$ & 16 & 9 & \\
\hline Anatomical site & & & 0.671 \\
\hline Distal femur & 16 & 11 & \\
\hline Proximal tibia & 5 & 1 & \\
\hline Other & 5 & 3 & \\
\hline Histological type & & & 0.743 \\
\hline Osteoblastic & 16 & 10 & \\
\hline Other & 10 & 5 & \\
\hline Enneking classification & & & 0.002 \\
\hline IA & 0 & 2 & \\
\hline IB & 0 & 3 & \\
\hline IIA & 3 & 6 & \\
\hline IIB & 22 & 4 & \\
\hline III & 1 & 0 & \\
\hline
\end{tabular}

A significant negative correlation was identified between the immunohistochemical staining scores and Enneking classification results of the 41 osteosarcoma specimens. ING4, inhibitor of growth 4 .

$2(18.2 \%)$ normal bone specimens (Fig. 2A) and a score of $(+++)$ in $9(81.8 \%)$ specimens (Fig. 2C). Negative controls were used for all normal bone specimens (Fig. 2B and D). Immunohistochemical staining scores of (-) and (+) were not observed in the normal bone specimens. A significant difference was observed between ING4 immunohistochemical staining scores in the osteosarcoma and normal bone tissues $(\mathrm{P}<0.001)$ (Table I).

According to the Enneking classification system for malignant bone tumors $(35,36), 2$ osteosarcoma specimens with a score of $(+++)$ were classified as IA; 3 osteosarcoma specimens, including a score of $(++)$ in 2 and $(+++)$ in 1 , were classified as IB; 9 osteosarcoma specimens, including a score of $(+)$ in 3 and $(++)$ in 6 , were classified as IIA; 26 osteosarcoma specimens, including a score of (-) in $6,(+)$ in 16 , $(++)$ in 3 and $(+++)$ in 1 , were classified as IIB; and only 1 osteosarcoma specimen with a score of (-) was classified as III. No statistical significance was observed between gender, age at diagnosis, anatomical site or histological type and immunohistochemical staining of ING4. However, a significant negative correlation was identified between the immunohistochemical staining scores and Enneking classification results of the 41 osteosarcoma specimens $(\mathrm{P}=0.002)$ (Table II). 


\section{Discussion}

Previous studies have demonstrated that ING4 expression is suppressed or reduced in a number of malignancies, and the degree of expression is associated with the tumor grade $(11,18,20,28-31)$. To the best of our knowledge, no study has investigated the level of expression of ING4 in human osteosarcoma tissue as compared with normal bone tissue, or the prognostic value of ING4 expression in patients diagnosed with osteosarcoma.

Similar to the results of previous studies regarding other malignant tumors $(11,18,20,28-31)$, the results of the present study demonstrated that the osteosarcoma tissues had significantly decreased expression of ING4 when compared with the normal bone tissues $(\mathrm{P}<0.001)$. The majority of the osteosarcoma specimens had a score of $(-)$ or $(+)$, whilst no normal bone specimens had a score of (-) or $(+)$. However, the mean age of the 11 patients from whom the normal bone specimens were obtained (59 years old) was markedly higher than that of the 41 patients with osteosarcoma ( 31 years old). This may be because the 11 normal bone tissues were obtained from patients who underwent surgery following trauma. Data as to whether the patients had implicit or asymptomatic osteoporosis, which are highly prevalent in older individuals, could not obtained. However, to the best of our knowledge, there are currently no available studies that demonstrate a correlation between ING4 expression level in bone and potential osteoporosis. Consequently, the results of the present study are considered to be reliable and objective.

In the present study, no significance was observed between gender, age at diagnosis, anatomical site or histological type and immunohistochemical staining of ING4. Instead, it was noted that the degree of reduction in ING4 expression correlated with the progression from low to high grades of osteosarcoma, according to the Enneking classification system for malignant bone tumors $(\mathrm{P}=0.002)$. ING4, a novel tumor suppressor of the ING family, has potential tumor-suppressing effects that are exerted through various signaling pathways, including tumorigenesis, cell cycle regulation, angiogenesis, cell apoptosis, DNA repair, migration and transcriptional regulation (10-27,37-43). These functions of ING4, which acts as an oncogene suppressor in numerous tumor types, have been identified repeatedly in vitro and in vivo (10-27,37-43). To the best of our knowledge, the present study is the first to investigate the immunoreactivity of ING4 in osteosarcoma tissue. Considering that Enneking classification system has prognostic implications for tumor invasion and metastasis, the findings of the current study indicate that ING4 may also serve a suppressive role in the tumorigenesis, invasion and soft tissue extension of osteosarcoma. A limited number of studies have investigated the tumor suppressor effect of ING4 in osteosarcoma. It was previously reported that adenovirus (Ad)-mediated ING4 gene transfer significantly induced growth inhibition and apoptosis in MG-63 human osteosarcoma cells, and intratumoral injections of Ad-ING4 in athymic nude mice bearing osteosarcoma tumors significantly inhibited osteosarcoma xenograft tumor growth (44). A different study indicated that ING4 could suppress osteosarcoma progression through mitochondrial and NF- $\mathrm{KB}$ signaling pathways (45). However, further research is required to determine the exact tumor suppressive mechanism of ING4 in osteosarcoma.

One limitation of the present study was that the overall survival data of the 41 patients could not be obtained. However, the Enneking grade of the osteosarcoma specimens at the time of diagnosis was obtained during TMA analysis. A significant negative correlation was observed between the ING4 immunohistochemical staining scores and Enneking grade. Therefore, this suggests that ING4 may be also negatively correlated with survival of patients with osteosarcoma.

In conclusion, the results of the present study indicate that ING4 expression may be a promising prognostic marker for patients with osteosarcoma. The significant negative correlation observed between ING4 expression and Enneking grade may provide insight into the mechanism of tumor progression in osteosarcoma, and additionally provide a potential target for novel therapeutic strategies, with the aim of improving the length and quality of life in patients with osteosarcoma.

\section{Acknowledgements}

The authors would like to thank Professor Xiangning Meng (Laboratory of Medical Genetics, Harbin Medical University, Harbin, China) for providing technical support.

\section{References}

1. Hansen MF: Genetic and molecular aspects of osteosarcoma. J Musculoskelet Neuronal Interact 2: 554-560, 2002.

2. Hayden JB and Hoang BH: Osteosarcoma: Basic science and clinical implications. Orthop Clin North Am 37: 1-7, 2006.

3. Link MP, Goorin AM, Miser AW, Green AA, PrattCB, Belasco JB, Pritchard J, Malpas JS, Baker AR, Kirkpatrick JA, et al: The effect of adjuvant chemotherapy on relapse-free survival in patients with osteosarcoma of the extremity. N Engl J Med 314: 1600-1606, 1986.

4. Eccles SA and Welch DR: Metastasis: Recent discoveries and novel treatment strategies. Lancet 369: 1742-1757, 2007.

5. Rodriguez NI, Hoots WK, Koshkina NV, Morales-Arias JA Arndt CA, Inwards CY, Hawkins DS, Munsell MF and Kleinerman ES: COX-2 expression correlates with survival in patients with osteosarcoma lung metastases. J Pediatr Hematol Oncol 30: 507-512, 2008.

6. Wang CJ, Yang D and Luo YW: Recombinant ING4 suppresses the migration of SW579 thyroid cancer cells via epithelial to mesenchymal transition. Exp Ther Med 10: 603-607, 2015.

7. Zhang H, Zhou X, Xu C, Yang J, Xiang J, Tao M and Xie Y: Synergistic tumor suppression by adenovirus-mediated ING4/PTEN double gene therapy for gastric cancer. Cancer Gene Ther 23: 13-23, 2016.

8. Yan A, Yang C, Chen Z, Li C and Cai L: miR-761 promotes progression and metastasis of non-small cell lung cancer by targeting ING4 and TIMP2. Cell Physiol Biochem 37: 55-66, 2015

9. Wu J, Zhu Y, Xu C, Xu H, Zhou X, Yang J, Xie Y and Tao M: Adenovirus-mediated p53 and ING4 gene co-transfer elicits synergistic antitumor effects through enhancement of p53 acetylation in breast cancer. Oncol Rep 35: 243-522, 2016.

10. Garkavtsev I, Kozin SV, Chernova O, Xu L, Winkler F, Brown E, Barnett GH and Jain RK: The candidate tumour suppressor protein ING4 regulates brain tumour growth and angiogenesis. Nature 428: 328-332, 2004.

11. Klironomos G, Bravou V, Papachristou DJ, Gatzounis G, Varakis J, Parassi E, Repanti M and Papadaki H: Loss of inhibitor of growth (ING-4) is implicated in the pathogenesis and progression of human astrocytomas. Brain Pathol 20: 490-497, 2010.

12. Li X, Zhang Q, Cai L, Wang Y, Wang Q, Huang X, Fu S, Bai J, Liu J, Zhang G and Qi J: Inhibitor of growth 4 induces apoptosis in human lung adenocarcinoma cell line A549 via Bcl-2 family proteins and mitochondria apoptosis pathway. J Cancer Res Clin Oncol 135: 829-835, 2009. 
13. Liu Y, Yu L, Wang Y, Zhang Y, Wang Y and Zhang G: Expression of tumor suppressor gene ING4 in ovarian carcinoma is correlated with microvessel density. J Cancer Res Clin Oncol 138: 647-655, 2012.

14. Nozell S, Laver T, Moseley D, Nowoslawski L, De Vos M, Atkinson GP, Harrison K, Nabors LB and Benveniste EN: The ING4 tumor suppressor attenuates NF-kappaB activity at the promoters of target genes. Mol Cell Biol 28: 6632-6645, 2008.

15. Xie YF, Sheng W, Xiang J, Zhang H, Ye Z and Yang J: Adenovirus-mediated ING4 expression suppresses pancreatic carcinoma cell growth via induction of cell-cycle alteration, apoptosis and inhibition of tumor angiogenesis. Cancer Biother Radiopharm 24: 261-269, 2009.

16. Shiseki M, Nagashima M, Pedeux RM, Kitahama-Shiseki M, Miura K, Okamura S, Onogi H, Higashimoto Y, Appella E, Yokota J and Harris CC: p29ING4 and p28ING5 bind to p53 and p300 and enhance p53 activity. Cancer Res 63: 2373-2378, 2003.

17. Zhang $\mathrm{X}, \mathrm{Xu}$ LS, Wang ZQ, Wang KS, Li N, Cheng ZH, Huang SZ, Wei DZ and Han ZG: ING4 induces G2/M cell cycle arrest and enhances the chemosensitivity to DNA-damage agents in HepG2 cells. FEBS Lett 570: 7-12, 2004.

18. Li Z, Xie Y, Sheng W, Miao J, Xiang J and Yang J: Tumor-suppressive effect of adenovirus-mediated inhibitor of growth 4 gene transfer in breast carcinoma cells in vitro and in vivo. Cancer Biother Radiopharm 25: 427-437, 2010.

19. $\mathrm{Li} \mathrm{J}$ and $\mathrm{Li} \mathrm{G}$ : Cell cycle regulator ING4 is a suppressor of melanoma angiogenesis that is regulated by the metastasis suppressor BRMS1. Cancer Res 70: 10445-10453, 2010.

20. Cai L, Li X, Zheng S, Wang Y, Wang Y, Li H, Yang J and Sun J: Inhibitor of growth 4 is involved in melanomagenesis and induces growth suppression and apoptosis in melanoma cell line M14. Melanoma Res 19: 1-7, 2009.

21. Li X, Cai L, Chen H, Zhang Q, Zhang S, Wang Y, Dong Y, Cheng $\mathrm{H}$ and Qi J: Inhibitor of growth 4 induces growth suppression and apoptosis in glioma U87MG. Pathobiology 76: 181-192, 2009.

22. Li X, Cai L, Liang M, Wang Y, Yang J and Zhao Y: ING4 induces cell growth inhibition in human lung adenocarcinoma A549 cells by means of Wnt-1/beta-catenin signaling pathway. Anat Rec (Hoboken) 291: 593-600, 2008.

23. Hung T, Binda O, Champagne KS, Kuo AJ, Johnson K, Chang HY, Simon MD, Kutateladze TG and Gozani O: ING4 mediates crosstalk between histone $\mathrm{H} 3 \mathrm{~K} 4$ trimethylation and $\mathrm{H} 3$ acetylation to attenuate cellular transformation. Mol Cell 33: 248-256, 2009

24. Ozer A and Bruick RK: Regulation of HIF by prolyl hydroxylases: Recruitment of the candidate tumor suppressor protein ING4. Cell Cycle 4: 1153-1156, 2005.

25. Ozer A, Wu LC and Bruick RK: The candidate tumor suppressor ING4 represses activation of the hypoxia inducible factor (HIF). Proc Natl Acad Sci USA 102: 7481-7486, 2005.

26. Kim S, Chin K, Gray JW and Bishop JM: A screen for genes that suppress loss of contact inhibition: Identification of ING4 as a candidate tumor suppressor gene in human cancer. Proc Natl Acad Sci USA 101: 16251-16256, 2004.

27. Shen JC, Unoki M, Ythier D, Duperray A, Varticovski L, Kumamoto K, Pedeux R and Harris CC: Inhibitor of growth 4 suppresses cell spreading and cell migration by interacting with a novel binding partner, liprin alpha1. Cancer Res 67: 2552-2558, 2007.

28. Fang F, Luo LB, Tao YM, Wu F and Yang LY: Decreased expression of inhibitor of growth 4 correlated with poor prognosis of hepatocellular carcinoma. Cancer Epidemiol Biomarkers Prev 18: 409-416, 2009.
29. Li J, Martinka M and Li G: Role of ING4 in human melanoma cell migration, invasion and patient survival. Carcinogenesis 29: 1373-1379, 2008.

30. Li M, Jin Y, Sun WJ, Yu Y, Bai J, Tong DD, Qi JP, Du JR, Geng JS, Huang Q, et al: Reduced expression and novel splice variants of ING4 in human gastric adenocarcinoma. J Pathol 219: 87-95, 2009.

31. Wang QS, Li M, Zhang LY, Jin Y, Tong DD, Yu Y, Bai J, Huang Q, Liu FL, Liu A, et al: Down-regulation of ING4 is associated with initiation and progression of lung cancer. Histopathology 57: 271-281, 2010.

32. Borkosky SS, Gunduz M, Beder L, Tsujigiwa H, Tamamura R, Gunduz E, Katase N, Rodriguez AP, Sasaki A, Nagai N and Nagatsuka H: Allelic loss of the ING gene family loci is a frequent event in ameloblastoma. Oncol Res 18: 509-518, 2010.

33. Kim S, Welm AL and Bishop JM: A dominant mutant allele of the ING4 tumor suppressor found in human cancer cells exacerbates MYC-initiated mouse mammary tumorigenesis. Cancer Res 70: 5155-5162, 2010.

34. Mastroleo I: Post-trial obligations in the Declaration of Helsinki 2013: Classification, reconstruction and interpretation. Dev World Bioeth Oct 19, 2015 (Epub ahead of print).

35. Enneking WF, Spanier SS and Goodman MA: A system for the surgical staging of musculoskeletal sarcoma. Clin Orthop Relat Res (153): 106-120, 1980.

36. Enneking WF: A system of staging musculoskeletal neoplasms. Clin Orthop Relat Res (204): 9-24, 1986.

37. Mathema VB and Koh YS: Inhibitor of growth-4 mediates chromatin modification and has a suppressive effect on tumorigenesis and innate immunity. Tumour Biol 33: 1-7, 2012.

38. Mao ZL, He SB, Sheng WH, Dong XQ and Yang JC: Adenovirus-mediated ING4 expression reduces multidrug resistance of human gastric carcinoma cells in vitro and in vivo. Oncol Rep 30: 2187-2194, 2013.

39. Byron SA, Min E, Thal TS, Hostetter G, Watanabe AT, Azorsa DO, Little TH, Tapia C and Kim S: Negative regulation of NF- $\kappa \mathrm{B}$ by the ING4 tumor suppressor in breast cancer. PLoS One 7: e46823, 2012.

40. Han Z, Zhou C, Sun B, Yan Q and Zhang J: Experimental studies on the inhibition of adenovirus-ING4-OSM therapy on nasopharyngeal carcinoma proliferation in vitro and in vivo. Cell Biochem Biophys 70: 1573-1578, 2014.

41. Lou C, Jiang S, Guo X and Dong XS: ING4 is negatively correlated with microvessel density in colon cancer. Tumour Biol 33: 2357-2364, 2012.

42. Li S, Fan T, Liu H, Chen J, Qin C and Ren X: Tumor suppressor ING4 overexpression contributes to proliferation and invasion inhibition in gastric carcinoma by suppressing the $N F-\kappa B$ signaling pathway. Mol Biol Rep 40: 5723-5732, 2013.

43. Wei Q, He W, Lu Y, Yao J and Cao X: Effect of the tumor suppressor gene ING4 on the proliferation of MCF-7 human breast cancer cells. Oncol Lett 4: 438-442, 2012.

44. Xu M, Xie Y, Sheng W, Miao J and Yang J: Adenovirus-mediated ING4 gene transfer in osteosarcoma suppresses tumor growth via induction of apoptosis and inhibition of tumor angiogenesis. Technol Cancer Res Treat 14: 369-378, 2015.

45. Li M, Zhu Y, Zhang H, Li L, He P, Xia H, Zhang Y and Mao C: Delivery of inhibitor of growth 4 (ING4) gene significantly inhibits proliferation and invasion and promotes apoptosis of human osteosarcoma cells. Sci Rep 4: 7380, 2014. 